\title{
Spin magnetotransport in a two-dimensional electron system confined in a quantum well
}

\author{
Roberto Cangas $^{1}$ and Miguel Angel Hidalgo ${ }^{2}$ \\ ${ }^{1}$ Departamento de Física, Escuela Técnica de Ingeniería Industrial, Universidad Politécnica de Madrid, \\ Spain \\ ${ }^{2}$ Departamento de Física, Universidad de Alcalá, Alcalá de Henares (Madrid), Spain \\ E-mail: roberto.cangas@upm.es and miguel.hidalgo@uah.es
}

Received 23 May 2011, in final form 29 July 2011

Published 14 September 2011

Online at stacks.iop.org/SST/26/105016

\begin{abstract}
We show a simple model that computes the magnetoconduction in a two-dimensional electron system (2DES) where the spin is another degree of freedom in the system. The 2DES is confined in a quantum well $(\mathrm{QW})$ immersed in a heterostructure, where the Rashba spin-orbit interaction is present. When an external magnetic field is applied to the system, the competition between the spin-orbit interaction and the Zeeman effect on the magnetoconduction of the 2DES is analysed, in the cases where one or two sub-bands are occupied in the QW. In the model different spin-oriented 2DES can be treated independently, with a spin current associated with each system. The model has been tested with experimental results obtained from a 2DES formed in an InGaAs layer.
\end{abstract}

(Some figures in this article are in colour only in the electronic version)

\section{Introduction}

The advances in the fabrication of mesoscopic systems with few impurities and defects lead to the macroscopic observation of microscopic quantum effects, such as the quantum Hall effect (QHE) and Subnikov-de Haas (SdH) oscillations [1]. These phenomena are related to the magnetoconductance of charged particles in a two-dimensional electron system (2DES), where the spin of the charge carriers plays a relevant role, and is responsible for the appearance of even and odd plateaux in the integer QHE. It is also possible to manipulate not only the charge current in the devices, but also the spin of the carriers by means of magnetic and/or electric fields. In fact, in 1990, Datta and Das [2] proposed a spin-polarized field effect transistor (FET). The gate electrode on the top of the FET device is used to control, by means of an electric field, the spin of the electrons. This electric field induces a spin-orbit interaction (SOI) that breaks the spin degeneration of the energy states in the 2DES. Even without any external magnetic/electric field, the carriers of the 2DES are also spin polarized by the internal built-in electric field due the structure inversion asymmetry (SIA) of the semiconductor heterostructure. The first theoretical study of this effect was made by Rashba [3] in 1960 (the SOI due to SIA is called the Rashba effect). In 1989 Das et al obtained evidence of spin splitting carrier populations at zero magnetic fields in InGaAs/ InAlAs heterostructures [4]. Additionally, zinc-blende-type semiconductors have bulk inversion asymmetry (BIA). Due to this asymmetry the local electric field varies along the crystal directions and therefore the SOI [5] (Dresselhaus effect). More recent devices are proposed by Schliemann et al [6] and Nitta et al [7], both based on the spin manipulation by means of an electric field. Schielemann has proposed a spin-field-effect transistor based on SOI of both SIA and BIA types, where the spin-independent scattering processes have no influence on the spin transport, and also showed how the interplay between SIA and BIA can lead to k-independent spin wave functions. Nitta has proposed a device based on the interference of spinning currents guided in narrow wire rings.

This work analyses the electrical magnetoconductance (magnetoresistance) behaviour of a 2DES confined in a heterostructure quantum well $(\mathrm{QW})$, under $\mathrm{QHE}$ conditions, and with Rashba SOI effect (at low temperature) using a simple model based on semiconsiderations and taking into 
account the spin orientation degree of freedom. From the theoretical point of view several attempts to understand $\mathrm{SdH}$ magnetoconductance oscillations and the integer QHE have been published. The most accepted one is based on the 'gendanken' experiment thought up by Laughlin [8], where the 2DES-localized states due to ionized impurities and defects play a crucial role to explain the plateaux of the Hall affect and the $\mathrm{SdH}$ oscillations of the magnetoconductivity, with minima values close to zero. However, experimental evidence shows that the measures made on the 2DES with higher electron mobility (materials with few defects and impurities) provide better plateaux precision. The model that we proposed does not use localized states to explain the QHE and SdH effects, but a simple one-electron theory with two assumptions: first, the existence of a flow of carriers from/to the QW to/from the heterostructure where it is immersed (the heterostructure behaves as a 'source/drain' of charges), and where long relative variations in the 2DES carrier concentration occur with negligible variations in the $3 \mathrm{D}$ carriers density of the environment; second, external magnetic fields and/or SOI lifts the spin degeneration, splitting the 2DES into two independent 2DESs, one with parallel spin and the other with antiparallel one $[9,10]$. The first assumption indicates a constant value of Fermi level in the 2DES when the 2D carrier concentration changes when the applied magnetic field varies. The second assumption leads us to consider the electron system as the sum of two 2DES independent spin subsystems.

In the present work we consider a QW where two sub-bands are filled; hence we consider four 2DESs (two subsystems in each sub-band according to the two spin states). In order to obtain the magnetoresistivity of such a system, in section 2 we compute the energy of the carriers immersed in an external magnetic field perpendicular to the 2DES plane, and subject to SOI. Then we develop the density of states (DOS) corresponding to the four 2DESs confined in the QW described above. In section 3 we calculate the whole density of carriers and the corresponding at the Fermi level in every 2DES. Adding the four magnetoconductivities we determine the total magnetoconductivity and hence the magnetoresistivity. This model also determines accurately the integer QHE [10], obtaining the plateaux and zeros of the Hall and diagonal magnetoresistances respectively.

\section{Density of states}

The Hamiltonian of a 2DES confined in the $(x, y)$ plane, when an external magnetic field is applied, and taking into account the SOI, can be written as $[11,12]$

$$
\begin{aligned}
H= & \frac{(\boldsymbol{p}+e \boldsymbol{A})^{2}}{2 m^{*}}+\frac{1}{2} g^{*} \mu_{B} B+\alpha\left(\sigma_{x} k_{y}-\sigma_{y} k_{x}\right) \\
& +\beta\left(\sigma_{x} k_{x}-\sigma_{y} k_{y}\right)
\end{aligned}
$$

where $\boldsymbol{k}$ is the electron wave vector, $\boldsymbol{p}$ the generalized momentum, $\boldsymbol{A}$ the magnetic potential vector, $\boldsymbol{B}$ the external magnetic field, $g^{*}$ the effective $g$-factor, $\mu_{B}$ the Bohr magneton and $\sigma_{i=x, y, z}$ are the Pauli matrices. The parameter $\alpha$ is related to SIA spin-orbit interaction (Rashba parameter), and $\beta$ is a parameter related to the intrinsic BIA spin orbit coupling
(Dresselhaus parameter). The Rashba effect dominates in the 2DES formed in semiconductors with lower gap energy [13-15]. It can find measured values of $\alpha$ between $2 \times$ $10^{-12} \mathrm{eVm}$ and $5 \times 10^{-11} \mathrm{eVm}$ for a 2DES confined in InGaAs/InAlAs heterostructures [16, 17] (alloys where it takes higher values). Also in the 2DES formed in MOSFET devices $\alpha$ can be tuned with the gate voltage [18]. The Dresselhaus $\beta$ parameter varies strongly with the crystal directions and with the width of the QW, obtaining values of $1.4 \times 10^{-13} \mathrm{eVm}$ for a $20 \mathrm{~nm}$ wide InGaAs-QW [13]. In QW and heterostructure devices made of InGaAs/InAlAs systems the SIA effect has more relevance than BIA, obtaining spin-split energies of the order of meV at Fermi level. Recent work shows a large Rashba spin splitting of $200 \mathrm{meV}$ in a 2DES confined in a metallic surface-state band on $\mathrm{Pb} / \mathrm{Ge}$ (111) at room temperature [19], opening new technological developments in semiconductor spintronic applications. We will focus this work on the 2DES only affected by SIA spinorbit interaction, which is controlled by an external electric field and hence the $\alpha$ parameter.

If the 2DES is confined in a QW with subbands' energy levels $E_{i}(i=1,2, \ldots)$, the eigenvalues of (1), assuming only the Rashba effect, are given by the expression $[3,11]$

$$
E_{i N_{L}}^{s}=E_{i}+\hbar \omega\left[N_{L}+s \frac{1}{2} \sqrt{\left(1-\left|g^{*}\right| \frac{m^{*}}{2 m_{0}}\right)^{2}+\frac{\gamma}{B} N_{L}}\right]
$$

with $s= \pm$ for $N_{L}=1,2,3, \ldots, s=+$ for $N_{L}=0, \gamma=$ $8 \alpha^{2} m^{* 2} / \hbar^{3} e, m_{0}$ is the free electron mass and $\omega=e B / m^{*}$. The states given by (1) are highly degenerate [11], with a degeneracy of $\left(2 \pi l^{2}\right)^{-1}$, where $l=\sqrt{\hbar /(e B)}$ is the magnetic length. In the limit of large magnetic fields the Zeeman term dominates the spin splitting, obtaining $\Delta E_{\text {spin }}=g^{*} \mu_{B} B$. In the opposite limit when $B \rightarrow 0, \Delta E_{\text {spin }}=2 \alpha k_{F}$ at the Fermi energy, where $k_{F}=\sqrt{2 \pi n_{0}}$ is the Fermi wave vector and $n_{0}$ the total 2DES carrier concentration. If we compare equation (2) with the spin-up and spin-down energy states associated with a conventional Landau level $N_{L}$, this corresponds to the $E_{N_{L}}^{+}$and $E_{N_{L}+1}^{-}$states, i.e. $\Delta E_{\text {spin }}=$ $\left|E_{N_{L}}^{+}-E_{N_{L}+1}^{-}\right|$.

At zero magnetic field, the SIA spin-split energy of a 2DES is $E_{ \pm}(k)=\mu_{c} k^{2} \pm \alpha k$ where $\mu_{c}=\hbar^{2} / 2 m$, and the density of states (DOS) of the spin-split branches at zero temperature has the form [12]

$$
\begin{aligned}
& D_{ \pm}(E)=\frac{1}{4 \pi \mu_{c}}\left[1 \mp \frac{\alpha}{\sqrt{4 \mu_{c} E+\alpha^{2}}}\right], \quad E \geqslant 0 \\
& D_{-}(E)=\frac{1}{2 \pi \mu_{c}} \frac{\alpha}{\sqrt{4 \mu_{c} E+\alpha^{2}}}, \quad E<0 .
\end{aligned}
$$

The DOS converges to the constant value $D_{0}=m^{*} / \pi \hbar^{2}$ (no spin degeneration is considered) when $\alpha$ is zero. In a QW with two filled subbands with energies $E_{1}$ and $E_{2}$, the $2 \mathrm{DES}$ can be considered as the sum of four 2DESs, everyone related to the $E_{1 \uparrow}, E_{1 \downarrow}, E_{2 \uparrow}$ and $E_{2 \downarrow}$ states. Hence the whole DOS of the four subsystems is computed by the expression

$$
D(E)=\sum_{s} \sum_{i} D_{i s}(E) .
$$




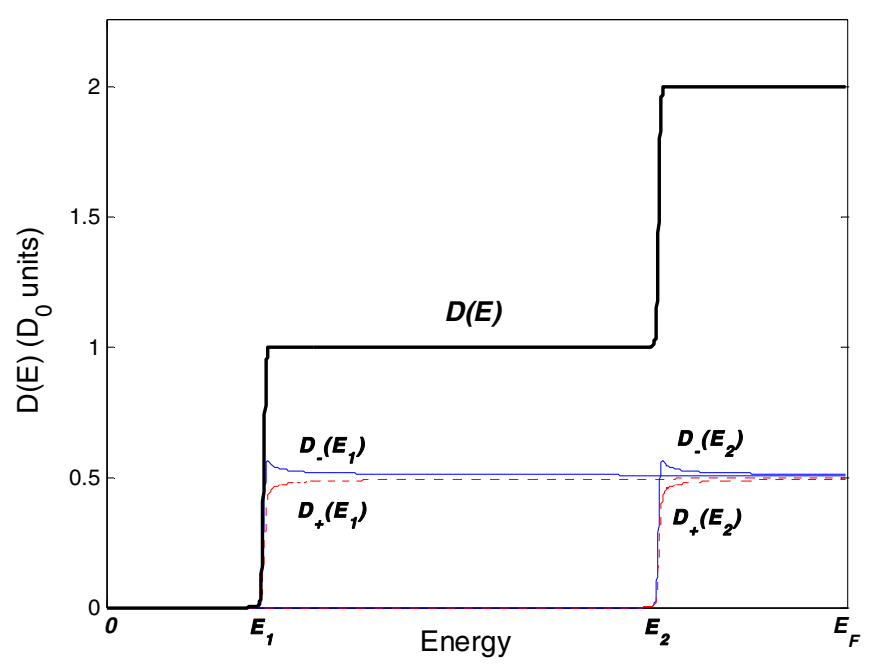

Figure 1. Density of states of a 2DES with Rashba SOI at zero external magnetic field. The whole DOS (black line) is the sum of the contributions of the two filled subbands with energies $E_{1}$ and $E_{2}$. Also each $E_{i}$ subband is split into two spin systems, with DOS $D\left(E_{i}\right)_{+}$and $D\left(E_{i}\right)_{-} . E_{F}$ is the Fermi level.

Figure 1 shows the DOS of the $2 \mathrm{DES}$ confined in a QW with two subbands (with energy leves $E_{1}$ and $E_{2}$ ). The whole density of states is considered as the sum of the four DOS independent related to the four 2DESs.

The DOS of a 2DES under the application of a magnetic field normal to the system has a shape like a 'comb', where the pinned 'teeth' are related to the $E_{N_{L}}^{s}$ values, and can be modelized with an 'ad hoc' Gaussian shape function [20]:

$$
\begin{gathered}
D(E)_{i s}=(e B / h) \sum_{s} \sum_{N_{L}}\left[(\pi / 2) \Gamma_{N_{L} s}^{2}\right]^{-1 / 2} \\
\times \exp \left\{-2\left(E-E_{i N_{L}}^{s}\right)^{2} / \Gamma_{N_{L} s}^{2}\right\} .
\end{gathered}
$$

The level broadening $\Gamma_{N_{L} s}$ is strongly dependent on the range of the scattering potentials. For short-range scatters $\Gamma_{N_{L} S}^{2}$ depends on the strength of the magnetic field. The broadening due to long-range potentials is proportional to the fluctuations of the local potential energy $\left((V(\mathbf{r})-\langle V(\mathbf{r})\rangle)^{2}\right.$, and can be considered negligible in samples where the impurities are far from the 2DES. Then, we consider only short-range scatters and use the expression [20] $\Gamma_{N_{L} s}=$ $\Gamma_{0}+\kappa \sqrt{\left(2 \hbar^{2} / \pi\right)(\omega / \tau)}$, where $\Gamma_{0}$ and $\kappa$ are fitting parameters, and $\tau$ is the relaxation time that takes into account the transport and spin relaxation processes. We assume the DyakonovPerel relaxation mechanism $[21,22]$ which describes the spin relaxation of free electrons.

Figures $2(a)-(f)$ show the evolution of the DOS presented in figure 1 when an external magnetic field is applied and exists the Rashba effect. To model the DOS we have used a Rashba parameter of $\alpha=0.7 \times 10^{-11} \mathrm{eVm}$ and an effective g-factor $g^{*}=4$. In order to compute the width of the Gaussian function of energy levels, we use the fitting parameters $\Gamma_{0}=0.01 E_{F}$, $\kappa=1$. The relaxation time is $\tau_{i s}=10^{-12} \mathrm{~s}$ given by Burg et al [23].

Figures 2(a) and $(b)$ show the oscillations and nodes of the DOS. The maxima and minima values of the oscillations occur when there is coincidence of the energy levels of the different spin 2DES, i.e. $D(E)$ have a maximum value when $E=E_{N_{L}}^{ \pm}=E_{N_{L}^{\prime}}^{\mp},\left(N_{L} \neq N_{L}^{\prime}\right)$ in every subband, and also when there is coincidence of the energy levels of the maxima values of the DOS in the two subbands, and at the same time the coincidence of the minima values in the oscillations. The nodes occur when there is no coincidence of energy states [9] in the DOS, i.e. when $E=E_{N_{L}}^{ \pm} \neq E_{N_{L}^{\prime}}^{\mp}$. The number of nodes and their position depend on the energy balance between Rashba and Zeeman terms. The Rashba term grows with the momentum, and hence with the energy, while the Zeeman term remains constant. The lower the magnetic field $B$, the larger the number of levels $N_{L}$, and more nodes can occur.

Figures 2(c) and (d) show with clarity the energy levels in both subbands. In figure $2(c)$ there is an overlapping of the $E_{i N_{L}}^{+}$and $E_{i N_{L}}^{-}$levels in the DOS in each subband and when both subbands are added. In figure $2(d)$ there is coincidence of energy levels of different spin in each subband, i.e. $E_{i N_{L}}^{ \pm}=E_{i N_{L}^{\prime}}^{\mp},\left(N_{L} \neq N_{L}^{\prime}\right)$, but there is no overlapping of the levels of the two subbands. Figures $2(d)$ and $(f)$ show the DOS at high magnetic fields ( $8 T$ and $12 T$ respectively). The height of the DOS levels depends again on the coindicence of levels intrasubband and the overlapping of levels intersubbands. As we will see below this DOS behaviour and its value at Fermi level explain the magnetoconductance of the $2 \mathrm{DES}$.

\section{Magnetoconduction}

When the applied magnetic field increases, the energy levels $E_{N_{L}}^{s}$ move to the Fermi level $\left(E_{F}\right)$, and the conduction occurs when each level crosses $E_{F}$, providing the SdH oscillation in the magnetoconductivity. The minimal values of the $\mathrm{SdH}$ oscillations occur when there is no coincidence between $E_{N_{L}}^{s}$ and $E_{F}$, and the maximal values occur when $E_{N_{L}}^{s}=E_{F}$. On the other hand, when two kinds of carriers are present in the system the $\mathrm{SdH}$ oscillations show a beating pattern behaviour. In a 2DES confined in semiconductor heterostructures the $\mathrm{SdH}$ beating pattern arises from the existence of two different 2DES, spin-up and spin-down electrons systems respectively [4].

In order to obtain the magnetoconductivity of the 2DES, formed in the semiconductor heterostructure, we have to calculate the density of carriers. Assuming that the 2DES is confined in a QW with two filled subbands, each subband energy level can be considered as a pocket that contains two independent 2DES, with spins parallel and antiparallel to the magnetic field. Therefore, the whole carrier concentration confined in the QW is given by the sum of the four 2DES concentrations:

$$
n=\sum_{s} \sum_{i} n_{i s}
$$

where again $i=1,2$ refers to each subband $E_{i}$, and $s$ refers to each spin orientation, and the carrier concentrations are obtained by the expressions $n_{i s}=\int_{-\infty}^{\infty} f_{0}(E) D_{i s}(E) \mathrm{d} E$. On 


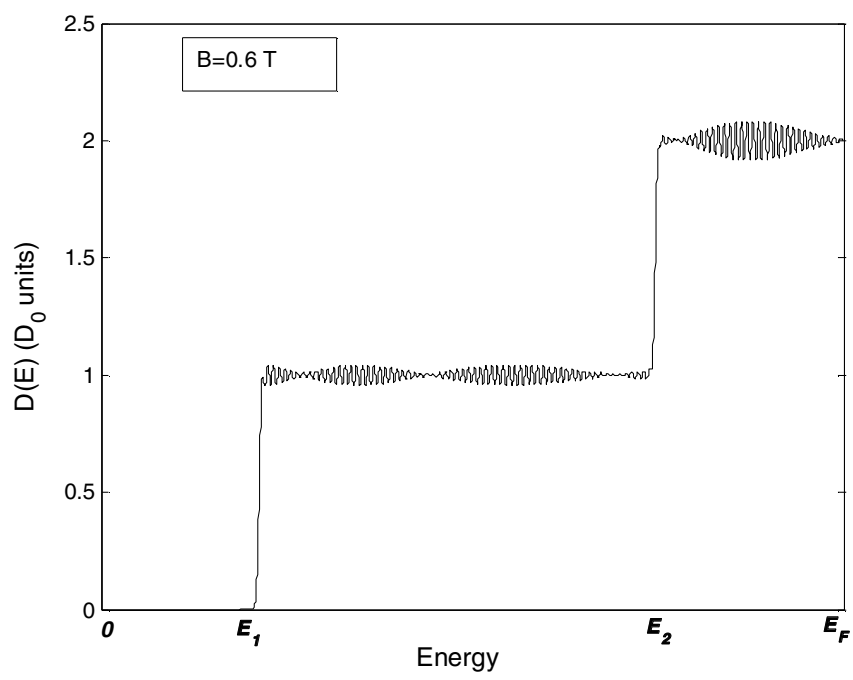

(a)

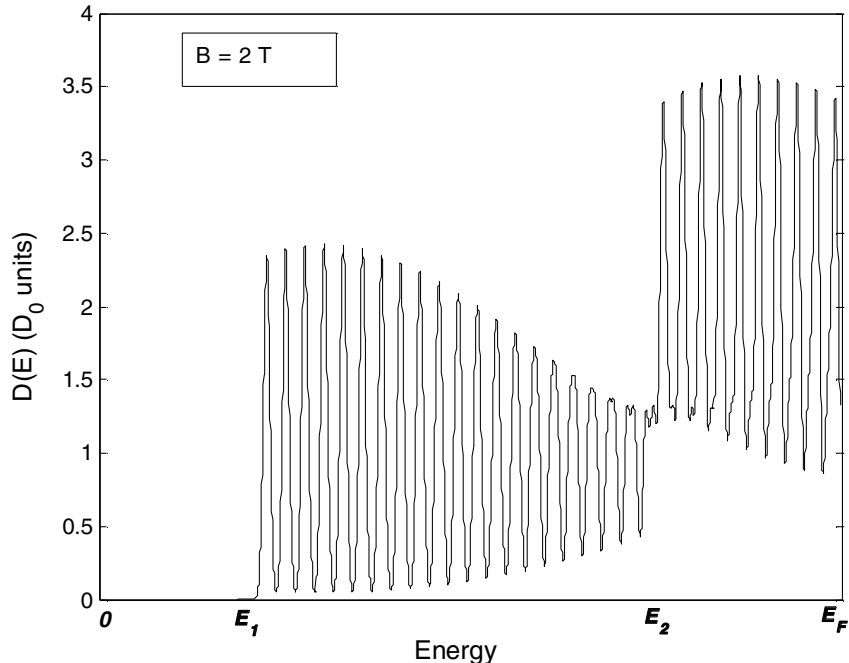

(c)

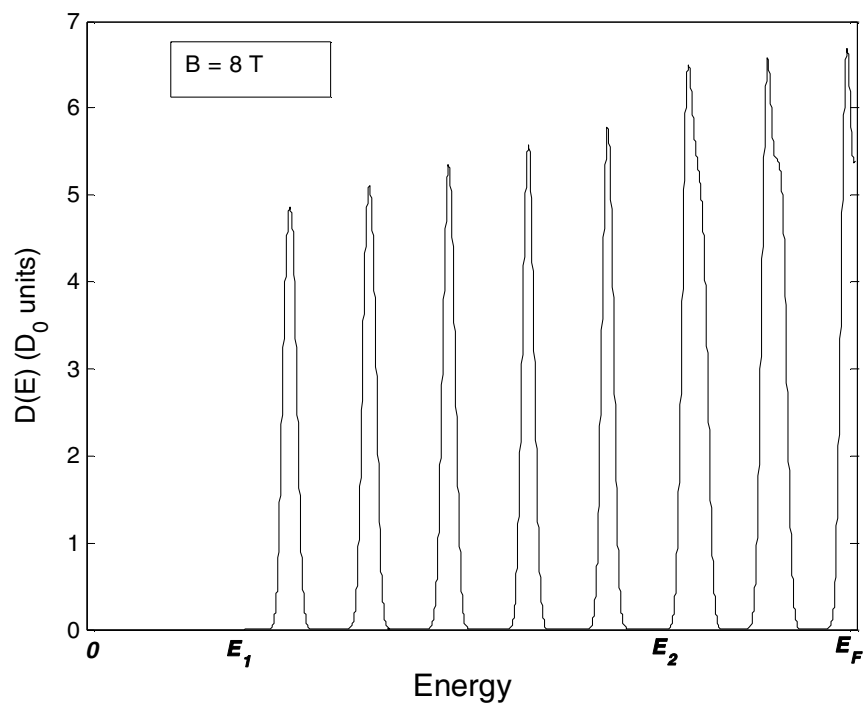

(e)

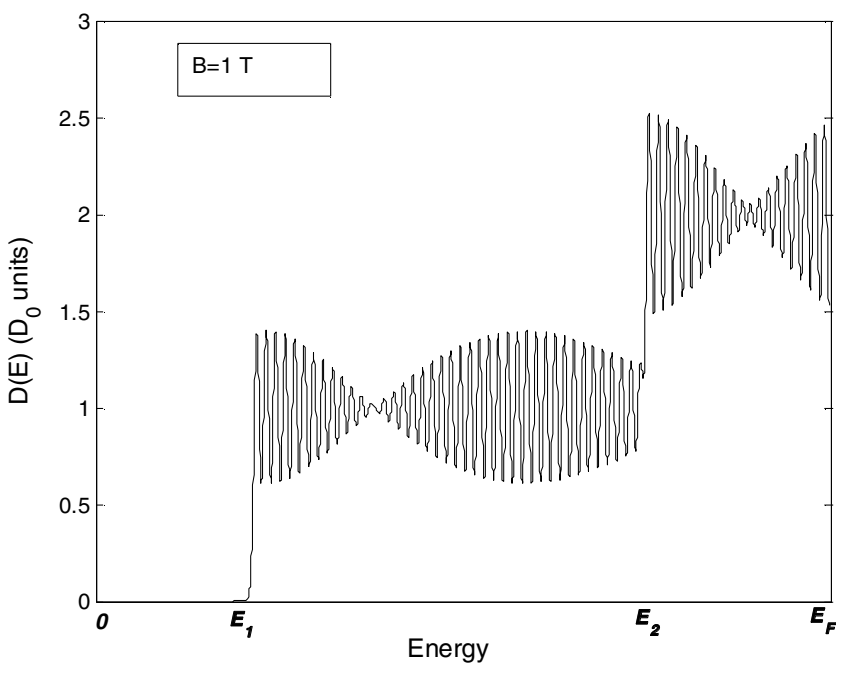

(b)

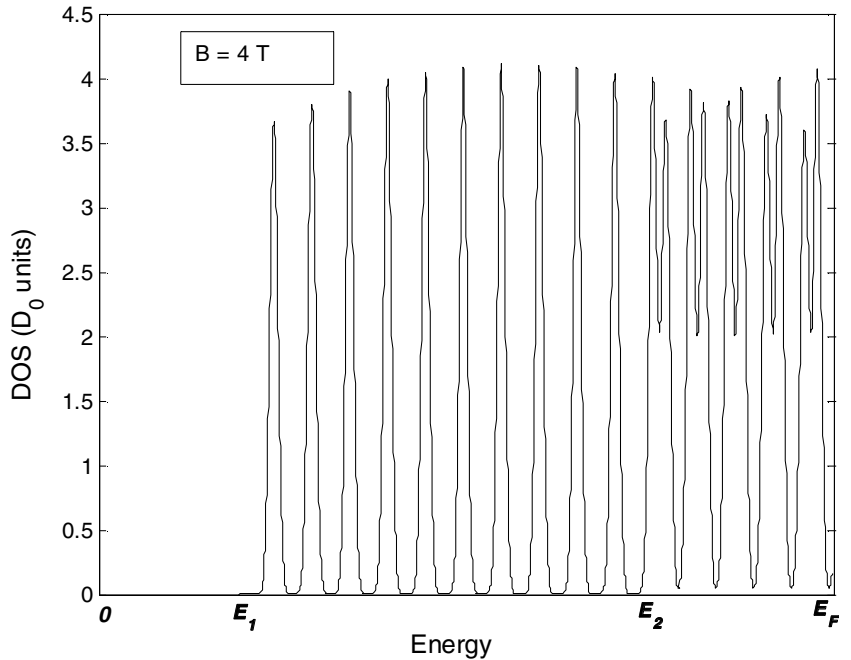

(d)

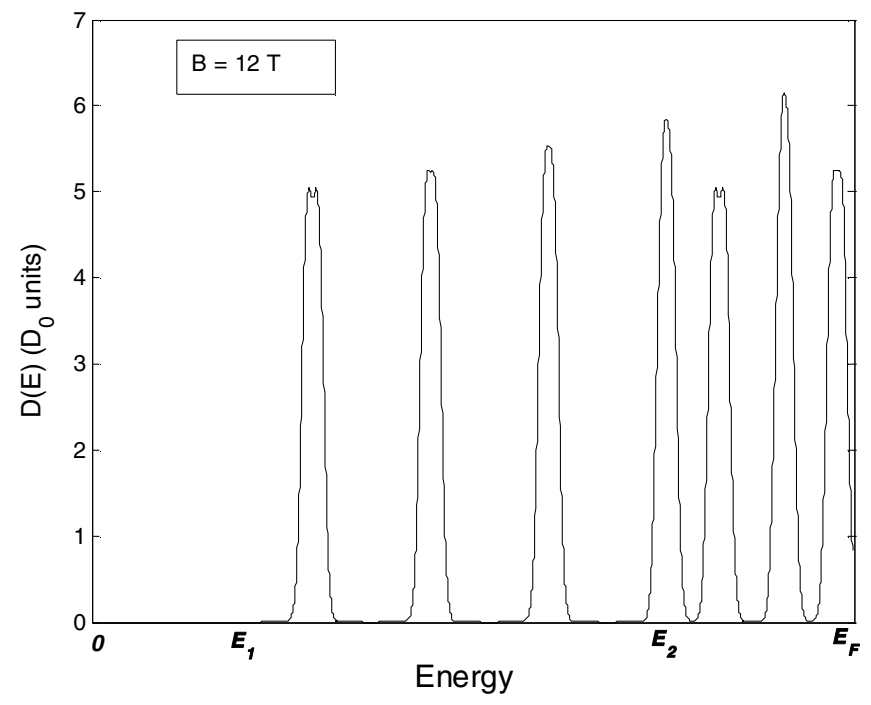

(f)

Figure 2. $(a)-(f)$ show the evolution of the density of states of a 2 DES when the magnetic field increases. The electron system is confined in a QW with two filled subbands. 


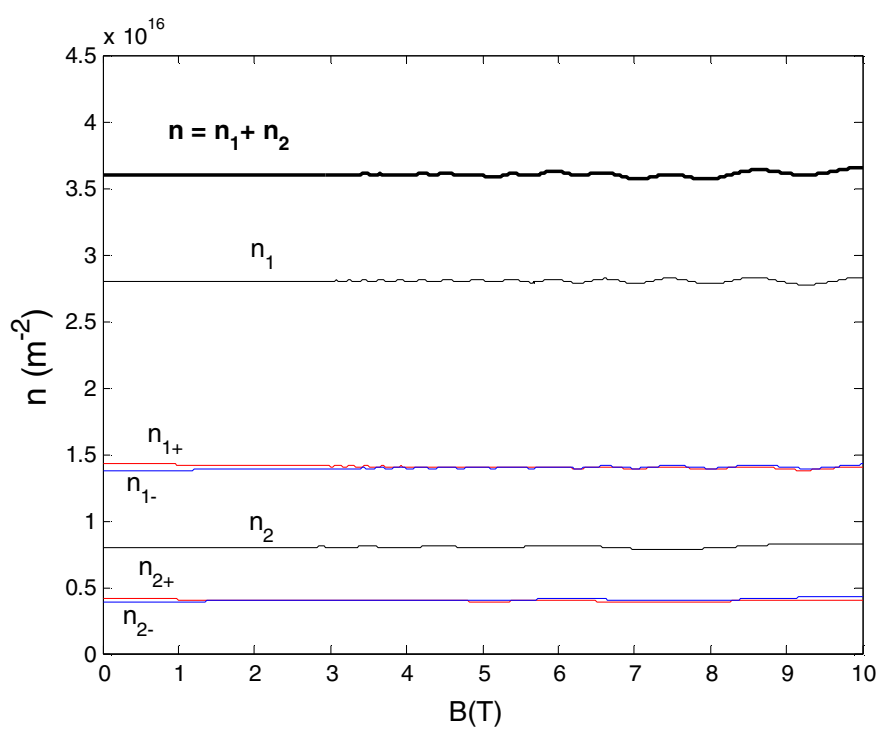

(a)

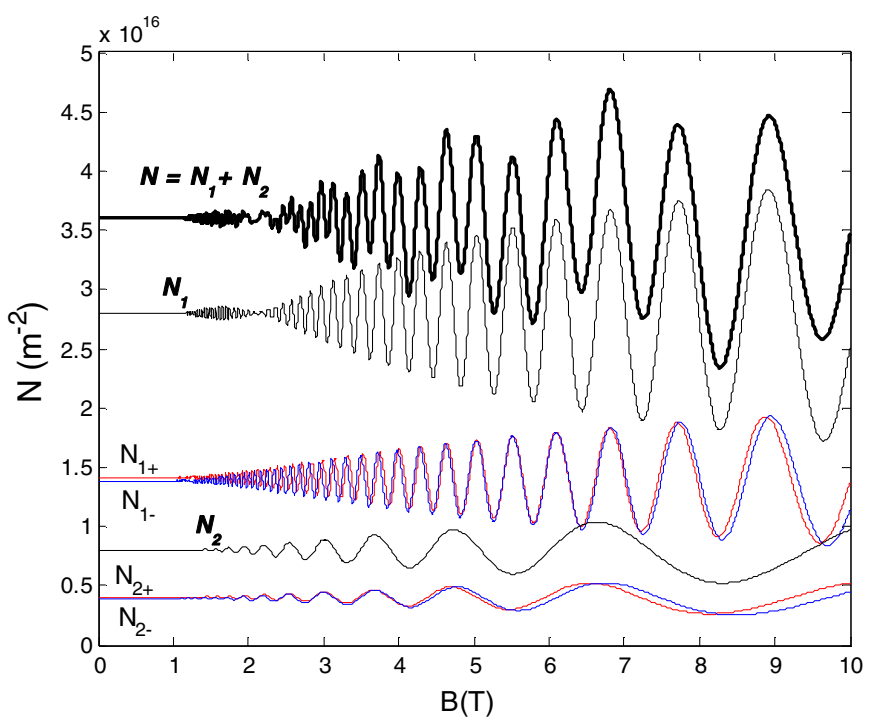

(b)

Figure 3. (a) Variation of the 2DES concentration with the magnetic field. (b) Variation of the 2DES carrier concentrations at Fermi level with the magnetic field. The red-dotted line and blue line are related to spin-up and spin-down orientations respectively. The black thin lines are related to carrier concentration at levels $E_{1}$ and $E_{2}$, and the black bold line is related to the whole 2DES carrier concentration.

the other hand the total carrier concentration $N$ at Fermi level $E_{F}$ is given by the expression

$$
N=\sum_{s} \sum_{i} N_{i s}
$$

where $N_{i s}=\int_{-\infty}^{\infty}\left(-\partial f_{0} / \partial E\right) D_{i s}(E) \mathrm{d} E$.

If the carrier concentration at zero external magnetic field is known, the Fermi level of the system is determined from equation (6). To compute the magnetoresistance we use experimental data obtained by Can Min Hu et al [24]. The 2DES is formed in a $20 \mathrm{~nm}$ thick $\mathrm{In}_{0.53} \mathrm{Ga}_{0.47}$ As layer where the two subbands levels are filled. The electron concentration at zero magnetic field is $n_{0}=3.6 \times 10^{16} \mathrm{~m}^{-2}$ and the carrier concentrations of the subbands are $n_{1}=$ $2.8 \times 10^{16} \mathrm{~m}^{-2}$ and $n_{2}=8 \times 10^{15} \mathrm{~m}^{-2}$. The calculated Fermi level is $E_{F}=0.172 \mathrm{eV}$ and the computed subband levels are $E_{1}=0.038 \mathrm{eV}$ and $E_{2}=0.134 \mathrm{eV}$. The effective mass is $0.05 m_{0}$. Figure $3(a)$ shows the evolution of the total carrier concentration in the whole 2DES $(n)$ when the external magnetic field increases, the evolution of the two subbands' carrier concentrations $\left(n_{1}\right.$ and $\left.n_{2}\right)$, and the evolution of the spin up/down 2DES that forms each subband $\left(n_{1+}, n_{1-}, n_{2+}, n_{2-}\right)$.

Figure $3(b)$ shows the evolution of the carrier concentration $N$ computed at Fermi Level in the whole 2DES, in the two subbands $\left(N_{1}\right.$ and $\left.N_{2}\right)$, and the evolution of the spin up/down 2DES that forms each subband $\left(N_{1+}, N_{1-}, N_{2+}, N_{2-}\right)$. The values of $N_{1}$ and $N$ show a beating pattern with a node near to $2.2 \mathrm{~T}$. The nodes occur when there is no coincidence of the levels $E_{N_{L}}^{+}$and $E_{N_{L}^{\prime}}^{-}$, at Fermi level. The $N$ value also shows an envelope modulation created by the sum of the $\mathrm{N}_{2}$ value.

Consider a competition between Zeeman and Rashba effects. Both effects cancel each other when $E_{N_{L}}^{+}=E_{N_{L}+1}^{-}=$ $E_{F}[10,17]$. In this case there is a coincidence of the value of the spin-up and spin-down carrier concentrations at Fermi level, i.e. the spin split energy is zero. We can see in figure $3(b)$ that this occurs at values of the magnetic field close to $3 \mathrm{~T}$ for the 2DES confined in the $E_{2}$ subband and $6 \mathrm{~T}$ for the 2DES confined in the $E_{1}$ subband, where $N_{i+}=N_{i-}$ in both subbands.

In the semiclassical approximation, when an electric field is applied (normal to external applied magnetic field), the carriers move with velocity $\mathbf{v}=\mathbf{v}_{\mathrm{d}}+\mathbf{v}_{\mathrm{c}}$, where $\mathbf{v}_{\mathrm{d}}$ is the drift velocity and $\mathbf{v}_{\mathrm{c}}$ the cyclotron one. We assume that the mean value $\left\langle\mathbf{v}_{\mathrm{c}}\right\rangle=0$ when the carriers move in the system and we use the Boltzmann distribution function for carriers perturbed by an electric and magnetic field [25, 26]. Taking into account the previous assumptions and using the linear relationship $\mathbf{j}=[\sigma] \mathbf{E}$, where $\mathbf{E}$ is the applied electric field and

$$
[\sigma]=\sum_{s} \sum_{i}[\sigma]_{i, s}
$$

which is the magnetoconductivity tensor, with components

$$
\begin{aligned}
& \sigma_{x x}=\sigma_{y y}=\sum_{s} \sum_{i}\left\{\left(e^{2} N_{i s} \tau_{i s} / m^{*}\right) /\left(1+\left(\omega \tau_{i s}\right)^{2}\right)\right\} \\
& \sigma_{x y}=-\sigma_{y x}=\sum_{s} \sum_{i}\left\{\left(e^{2} n_{i s} \tau_{i s} / m^{*}\right) \cdot \omega \tau_{i s} /\left(1+\left(\omega \tau_{i s}\right)^{2}\right)\right\}
\end{aligned}
$$

the current density for the two subband problem can be expressed:

$$
\mathbf{j}=[\sigma] \mathbf{E}=\left(\mathbf{j}_{1}+\mathbf{j}_{2}\right)_{\uparrow}+\left(\mathbf{j}_{1}+\mathbf{j}_{2}\right)_{\downarrow} .
$$

The magnetoresistivities are obtained by the relationship between tensors $[\rho]=[\sigma]^{-1}$, with components $\rho_{x x}=\rho_{y y}=$ $\sigma_{x x} /\left(\sigma_{x x}^{2}+\sigma_{x y}^{2}\right)$ and $\rho_{x y}=-\rho_{y x}=\sigma_{x y} /\left(\sigma_{x x}^{2}+\sigma_{x y}^{2}\right)$.

We reproduce the value of the magnetoresistivity given in reference [24] when the whole 2DES carrier concentration 


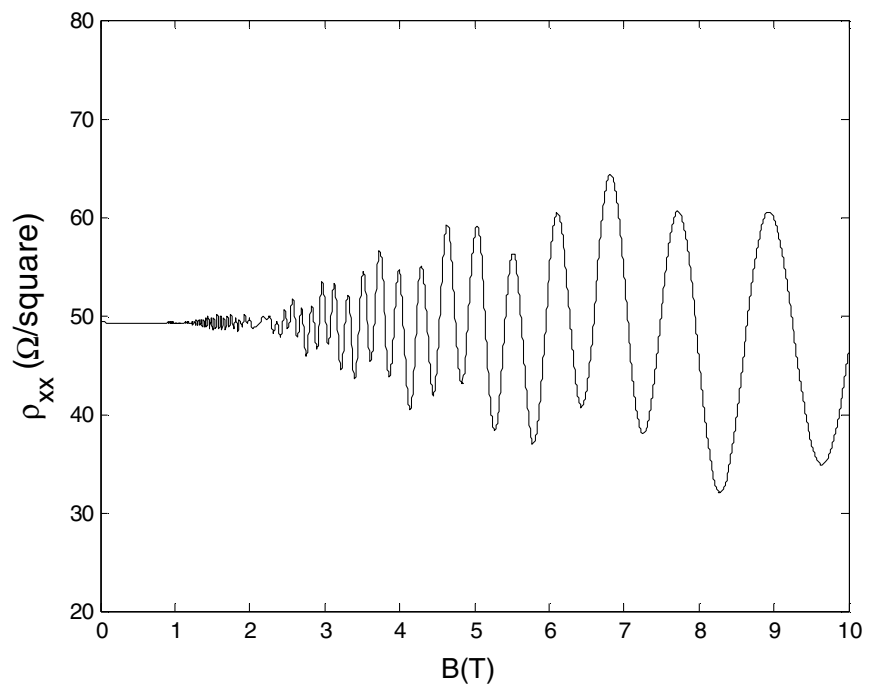

(a)

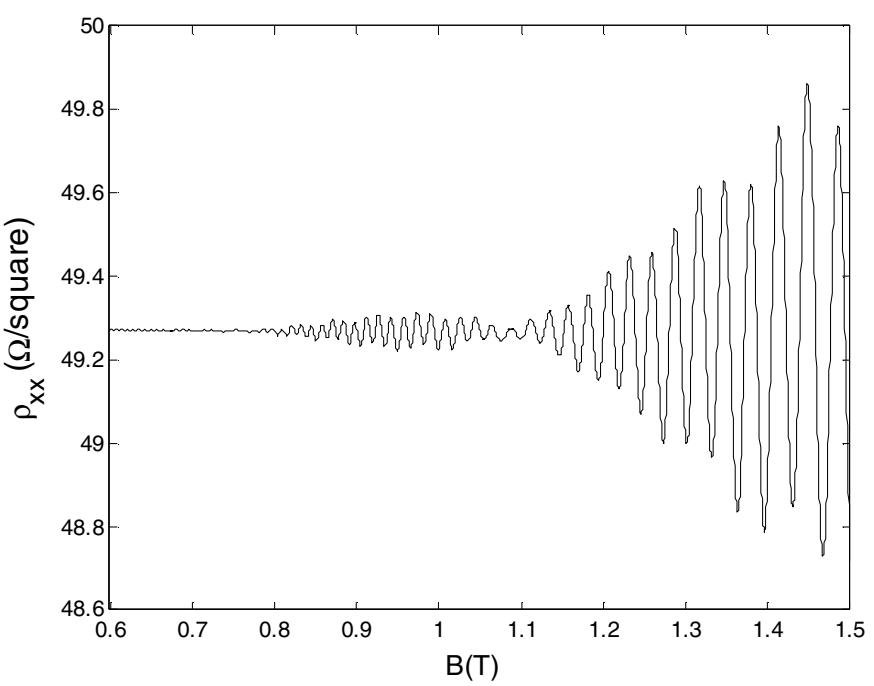

(b)

Figure 4. (a) SdH oscillations beating pattern of the magnetoresistivity, with a visible node in the region between $2 \mathrm{~T}$ and $2.5 \mathrm{~T}$. (b) Detailed plot of the $\mathrm{SdH}$ oscillations that shows a clean node at $1.1 \mathrm{~T}$, and another in the $0.7 \mathrm{~T}-0.8 \mathrm{~T}$ interval.

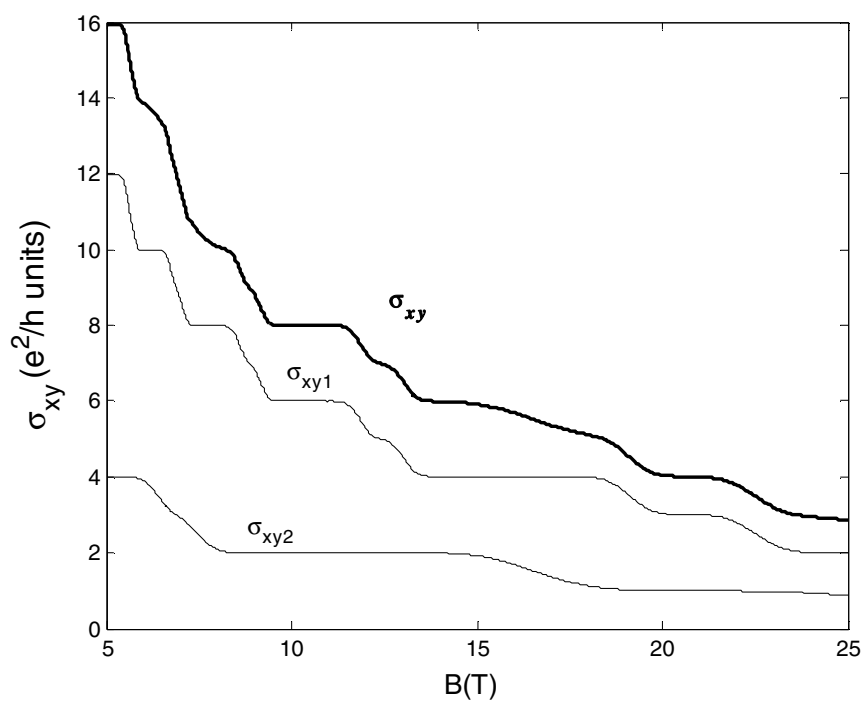

(a)

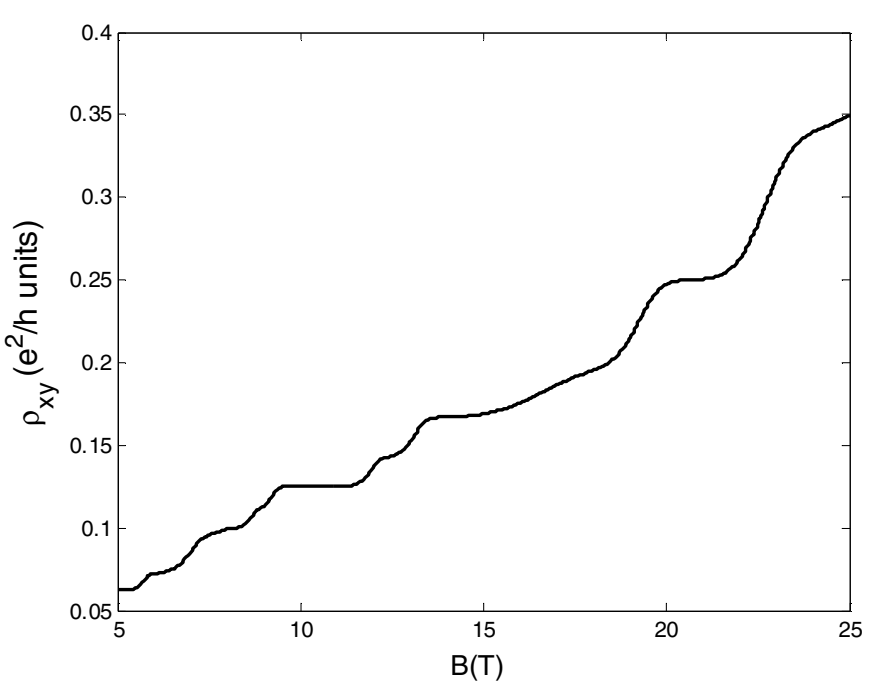

(b)

Figure 5. (a) Magnetoconductivity $\left|\sigma_{x y}\right|$ of the whole 2DES and magnetoconductivities $\left|\sigma_{x y i}\right|$ of the subsystems related to each filled subband versus the external applied magnetic field. $(b)$ Magnetoresistivity of the 2DES versus the external applied magnetic field.

is $3.6 \times 10^{16} \mathrm{~m}^{-2}$ and two energy subbands in the QW are filled. Figure $4(a)$ shows the $\mathrm{SdH}$ oscillations of the magnetoresisitivity with a visible node near to $2.2 \mathrm{~T}$, and figure $4(b)$ shows a detailed plot in the interval $0.6 \mathrm{~T}-$ $1.5 \mathrm{~T}$ of the magnetic field, where two more nodes at values near $0.75 \mathrm{~T}$ and $1.1 \mathrm{~T}$ respectively are also shown. As we said before, the nodes occur when there is no coincidence between energy levels at $E_{F}$, i.e. when $E_{N_{L}}^{+} \neq E_{N_{L}^{\prime}}^{-}$at Fermi level. The appearance and definition of the nodes depend on the overlapping and the width $\Gamma_{N_{L} s}$ of the DOS energy levels.

Figure 5(a) shows the calculated Hall magnetoconductivity $\left(\sigma_{x y}\right)$ of the whole 2DES and the Hall magnetoconductivities $\left(\sigma_{x y 1}, \sigma_{x y 2}\right)$ related to the two subbands (obtained with the experimental data [24] used above to compute the SdH oscillations). The behaviour of $\sigma_{x y 1}$ and $\sigma_{x y 2}$ correspond to the pattern of the integer quantum Hall effect each one, with plateaux that have values of magnetoconductivity equal to $v e^{2} / h, v=1,2,3, \ldots$. This is the result that we expect because each 2DES is treated independently, and the model reproduces the integer QHE in the case of only one filled subband [10]. The total Hall magnetoconductivity $\sigma_{x y}$ is the sum $\sigma_{x y 1}$ and $\sigma_{x y 2}$ and also has plateaux with values $v e^{2} / h$, although with less definition. Figure $5(b)$ shows the Hall magnetoresistivity of the 2DES, where one can observe plateaux with values of magnetoresistivity equal to $h /\left(v e^{2}\right)$. In both figures we have selected the interval of the magnetic field from 5 to $25 T$ in order to show well-resolved plateaux at low filling factors $v$. 


\section{Conclusions}

In conclusion, we have developed a simple semiclassical theory that reproduces the magnetoconduction of a $2 \mathrm{DES}$ confined in a QW when two subbands are occupied and when the competition between Rashba and Zeeman effects is significant. Then, in the model that we use, the spin plays an important role in the magnetoconduction. The model starts with the whole carrier concentration at zero external magnetic field, that establishes the Fermi level. When two subbands are occupied, the carrier concentration of each subband is obtained from the value of the subband energy level with respect to Fermi level. Each subband is considered as the sum of two independent 2DESs with different spin polarizations due to the Rahsba effect. Therefore, we consider the whole 2DES confined in a QW as two filled subbands, and hence four independent 2DESs. The evolution of the DOS with the external applied magnetic field explains the $\mathrm{SdH}$ oscillations and the integer QHE. The model can be generalized to systems with more than two filled subbands.

\section{References}

[1] Klitzing K V, Dorda G and Pepper M 1980 Phys. Rev. Lett. 45494
[2] Datta S and Das B 1990 Appl. Phys. Lett. 56665

[3] Rashba E I 1960 Fiz. Tverd. Tela 21224 Rashba E I 1960 Sov. Phys. Solid State 21109

[4] Das B et al 1989 Phys. Rev. B 391411

[5] Dresselhaus D 1955 Phys. Rev. 100580

[6] Schiliemann J et al 2003 Phys. Rev. Lett. 90146801

[7] Nitta J et al 2009 J. Appl. Phys. 105122402

[8] Laughlin R B 1981 Phys. Rev. B 235632

[9] Cangas R and Hidalgo M A 2009 Physica E 411306

[10] Hidalgo M A and Cangas R 2010 Physica E 421329

[11] Yang W and Chang K 2006 Phys. Rev. B 73045303

[12] Winkler R 2003 Spin-orbit coupling effects in two-dimensional electron and hole systems Springer Tracts Mod Phys 191 163-9

[13] Meier L et al 2007 Nat. Phys. 3650

[14] Ganichev S D et al 2004 Phys. Rev. Lett. 92256601

[15] de Andrada e Silva E A et al 1994 Phys. Rev. B 508523

[16] Grundle D 2000 Phys. Rev. Lett. 846074

[17] Yang C L et al 2006 Phys. Rev. Lett. 96186605

[18] Nitta J et al 1997 Phys. Rev. Lett. 781335

[19] Yaji Koichiro et al 2010 Nature communications 117

[20] Ando T et al 1982 Rev. Mod. Phys. 54437

[21] Dyakonov M I and Perel V I 1972 Sov. Phys. 133023

[22] Glazov M M et al 2010 Physica E 422157

[23] Burgt et al 1995 Phys. Rev. B 5212218

[24] Hu C M and Nitta J et al 1999 Phys. Rev. B 607736

[25] Kittel C 1987 Quantum Theory of Solids (New York: Wiley)

[26] Askerov B M 1994 Electron Transport Phenomena in Semiconductors (Singapore: World Scientific) 